\title{
Preface to the special issue on Cyberworlds 2014
}

\author{
Andrés Iglesias $^{1,2} \cdot$ Mikio Shinya $^{2} \cdot$ Akemi Galvez $^{1}$
}

Published online: 27 November 2015

(C) Springer-Verlag Berlin Heidelberg 2015

This special issue includes the extended versions of the papers selected from Cyberworlds 2014 conference, held in Santander, Spain, 6-8 October 2014 (see the URL: http://www.cw2014.unican.es for details). The eight selected papers reflect the great diversity of topics of Cyberworlds 2014 conference as well as the excellent level of its scientific contributions.

The first paper, "Interactive Multilevel Focus+Context Visualization Framework", by Mahmudul Hasan, Faramarz Samavati and Christian Jacob, presents the construction of an interactive multilevel focus+context visualization framework for the navigation and exploration of large-scale 2D and 3D images. This paper was awarded with the Cyberworlds 2014 Best Paper Award during the gala dinner ceremony, held at the Casino of Santander on October 6th 2014. Do not miss the exciting accompanying video, with very illustrative examples about the visualization tool prototype described in the paper.

Second and third papers were jointly awarded the Cyberworlds 2014 Distinguished Paper Award. The second paper, "Dynamic Social Formations of Pedestrian Groups Navigating and Using Public Transportation in a Virtual City", by Francisco Rojas, Fernando Tarnogol and Hyun-seung Yang, introduces a two-level steering system based on two classes (the group agent and the pedestrian agent) to simulate the

Andrés Iglesias

iglesias@unican.es

http://personales.unican.es/iglesias

1 Department of Applied Mathematics and Computational Sciences, E.T.S.I. Caminos, Canales y Puertos, University of Cantabria, Avda. de los Castros, s/n, 39005 Santander, Spain

2 Department of Information Science, Faculty of Science, Narashino Campus, Toho University, 2-2-1 Miyama, Funabashi 274-8510, Japan dynamic social formations of pedestrian groups navigating and using public transportation in a virtual city. The new approach improves the realism of the simulation of social groups evolving in virtual worlds with several interesting features (slot-locking, an optimized ray casting technique, pal social gestures, a new formation adjustment algorithm, and others).

The third paper, "Real-time EEG-based Emotion Monitoring Using Stable Features”, by Zirui Lan, Olga Sourina, Lipo Wang and Yisi Liu, proposes a novel real-time subjectdependent algorithm with more stable features for integration of human emotion recognition algorithms based on electroencephalogram (EEG) signals within human-computer interfaces without re-training. The algorithm was tested on five subjects with four emotions induced and two EEG sessions per day during eight consecutive days. It was also integrated with real-time applications "Emotional Avatar" and "Twin Girls".

The fourth paper "CUDA-Based Realtime Hand Gestures Interaction and Volume Data Visualization Using Leap Motion", by Junchen Shen, Yanlin Luo, Zhongke Wu, Yun Tian, QingQiong Deng and Mingquan Zhou, proposes a touchless interaction technique for volume visualization by using the Leap Motion sensor. The system is based on a set of natural gestures for unimanual and bimanual interaction with different tools to perform tasks in real world-like way. Comparative experiments with other existing techniques were conducted. They show that this method (especially the designed bimanual interaction) has a better performance with less completion time.

The fifth paper, "Animated Visualization of SpatialTemporal Trajectory Data for Air-Traffic Analysis", by Stefan Buschmann, Matthias Trapp and Jürgen Döllner, presents a technique for real-time animated visualization of massive trajectory data with applications to air-traffic analy- 
sis that enables interactive spatial-temporal filtering, generic mapping of trajectory attributes to geometric representations and appearance, and real-time rendering features. As a general-purpose visualization technique, it can be useful in many applications involving traffic movement data, georeferenced networks, or spatial-temporal data.

The sixth paper, "Example-Based Caricature Generation with Exaggeration Control”, by Wei Yang, Masahiro Toyoura, Jiayi Xu and Xiaoyang Mao, proposes a new examplebased automatic caricature generation system supporting the exaggeration of both the shape of facial components and the spatial relationships among such components, capturing the prominent features of the subjects.

The seventh paper "Applying Clonal Selection Theory to Data Fitting with Rational Bezier Curves", by Andrés Iglesias, Akemi Gálvez and Andreina Avila, proposes a new hybrid strategy for data fitting through rational Bézier curves. The approach is based on the hybridization of a previous technique based on artificial immune systems for global optimization of the least-squares objective function and the mesh adaptive search algorithm for local refinement of the best solution. The experimental results show that this new approach outperforms the previous one by orders of magnitude on a benchmark of 27 instances.

The eighth and last paper, "EnergyViz: An Interactive System for Visualization of Energy Systems", by Haleh Alemasoom, Faramarz Samavati, John Brosz and David Layzell, introduces Energy Viz, a visualization system that integrates several visualization techniques (including flow diagram representation to show energy flow, 3D interaction with low diagrams, and some features for simultaneous exploration of spatial and abstract information) with an interface for exploring time-varying, multi-attribute and spatial properties of a particular energy system. The system can be used by the public and policy makers to analyze energy-related issues for green environment and sustainable energy.

We are deeply grateful to The Visual Computer Editorin-Chief, Professor Nadia Magnenat-Thalmann, and all Editorial Staff for their continuing help and assistance during the whole process of these papers preparation. We also wish to thank the authors for their high-quality contributions and their great cooperation in preparing this special issue. We owe special thanks to the conference program vice-chairs, Profs. Norimasa Yoshida (Nihon University, Japan) and Michio Shiraishi (Toho University, Japan), the program committee members and reviewers for their hard work in reviewing the papers and making constructive comments and suggestions, which have substantially contributed to improving all the papers. They undoubtely did a great job!

We also thank the Computer Science Research Program of the Spanish Ministry of Economy and Competitiveness, Project TIN2012-30768, the University of Cantabria (Santander, Spain) and Toho University (Funabashi, Japan) for their support and all the facilities given to produce this special issue. Last, but certainly not least, we wish to thank the readers for their interest in this special issue and for supporting The Visual Computer. Many thanks to all of them!

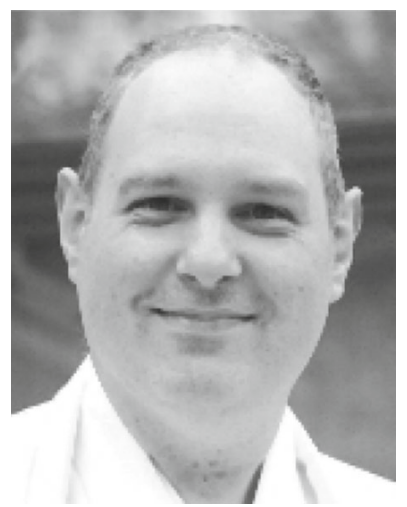

Andrés Iglesias is currently the Director of the Computer Graphics and Geometric Modeling research group at the University of Cantabria (Santander, Spain) and Visiting Professor of Toho University (Funabashi, Japan). He has published about 190 international papers in journals and conferences and 14 books. He has also been program committee member in more than 160 international conferences, reviewer of 60 journals (mostly ISI) and 150 conferences, and expert evaluator of several agencies such as NSF (USA), 7th. Framework Programme (EU), etc. His research interests are in the fields of computer graphics, geometric modeling, CAD/CAM, artificial intelligence, and soft computing.

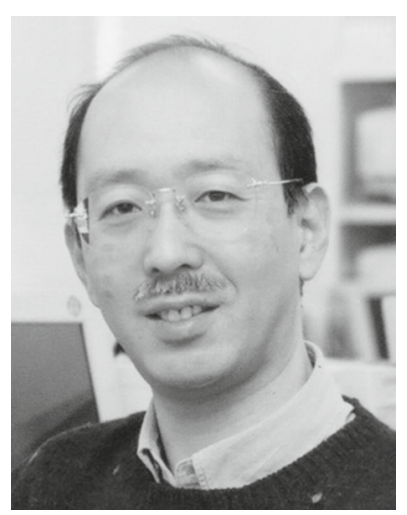

Mikio Shinya is currently a Professor at Department of Information Science, Toho University. He received a BSc in 1979 , an MS in 1981, and a PhD in 1990 from Waseda University. He joined NTT Laboratories in 1981, and moved to Toho University in 2001. He was also a visiting scientist at the University of Toronto in 1988-1989. His research interests include computer graphics and visual science.

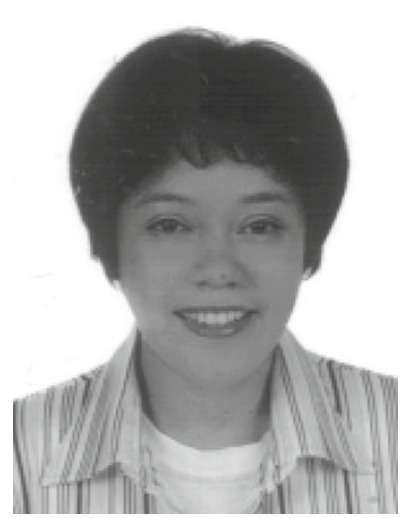

Akemi Galvez is currently Associate Professor at the Department of Applied Mathematics and Computational Sciences, University of Cantabria (Santander, Spain). She has published about 95 international papers about geometric modeling and processing, curve and surface reconstruction and soft computing in international journals and conferences. She is also member of the editorial board of some international journals, and program committee member of several international conferences on computational science. Her main research fields are computer-aided geometric design, geometric processing, curve and surface reconstruction, artificial intelligence and soft computing, and their industrial applications. 